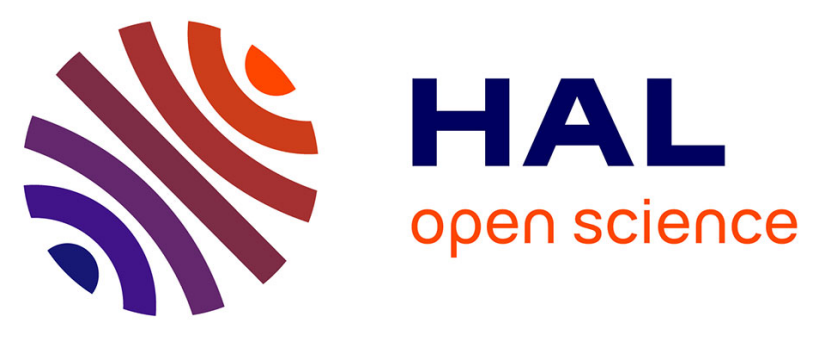

\title{
Modelling targets for anticancer drug control optimisation in physiologically structured cell population models
}

Frédérique Billy, Jean Clairambault, Olivier Fercoq, Tommaso Lorenzi, Alexander Lorz, Benoît Perthame

\section{To cite this version:}

Frédérique Billy, Jean Clairambault, Olivier Fercoq, Tommaso Lorenzi, Alexander Lorz, et al.. Modelling targets for anticancer drug control optimisation in physiologically structured cell population models. ICNAAM 2012 - 10th international conference of numerical analysis and applied mathematics, Simos, Thodoros, Sep 2012, Kos, Greece. pp.1323-1326, 10.1063/1.4756399 . hal-00780721

\section{HAL Id: hal-00780721 \\ https://hal.inria.fr/hal-00780721}

Submitted on 25 Jan 2013

HAL is a multi-disciplinary open access archive for the deposit and dissemination of scientific research documents, whether they are published or not. The documents may come from teaching and research institutions in France or abroad, or from public or private research centers.
L'archive ouverte pluridisciplinaire HAL, est destinée au dépôt et à la diffusion de documents scientifiques de niveau recherche, publiés ou non, émanant des établissements d'enseignement et de recherche français ou étrangers, des laboratoires publics ou privés. 


\title{
Modelling targets for anticancer drug control optimisation in physiologically structured cell population models
}

\author{
Frédérique Billy*, Jean Clairambault*, Olivier Fercoq ${ }^{\dagger}$, Tommaso Lorenzi**, \\ Alexander Lorz* and Benoît Perthame* \\ *INRIA Paris-Rocquencourt \& LJLL, Université Pierre-et-Marie-Curie, 4 Pl. Jussieu, 75005 Paris, France \\ ${ }^{\dagger}$ INRIA Saclay-Île-de-France \& CMAP, École Polytechnique, 91128 Palaiseau Cedex, France \\ ** Politecnico di Torino, Corso Duca degli Abruzzi, 24, 10129 Torino, Italy
}

\begin{abstract}
.
The main two pitfalls of therapeutics in clinical oncology, that limit increasing drug doses, are unwanted toxic side effects on healthy cell populations and occurrence of resistance to drugs in cancer cell populations.

Depending on the constraint considered in the control problem at stake, toxicity or drug resistance, we present two different ways to model the evolution of proliferating cell populations, healthy and cancer, under the control of anti-cancer drugs.

In the first case, we use a McKendrick age-structured model of the cell cycle, whereas in the second case, we use a model of evolutionary dynamics, physiologically structured according to a continuous phenotype standing for drug resistance.

In both cases, we mention how drug targets may be chosen so as to accurately represent the effects of cytotoxic and of cytostatic drugs, separately, and how one may consider the problem of optimisation of combined therapies.
\end{abstract}

Keywords: Cell population dynamics, Partial Differential Equations, Physiologically structured Models, Cell division cycle, Adaptive dynamics, Cancer, Drug control

PACS: 87.17.Aa, 87.17.Ee

\section{DRUG TARGETS IN PROLIFERATING CELL POPULATION DYNAMIC MODELS}

McKendrick physiological age-structured cell population dynamics models for the cell division cycle [1, 2, 3] describe the evolution of densities $n_{i}(t, x)$ of cells of age $x$ at time $t$ in phase $i$ of the cell cycle. Furthermore, one may include in such models targets for their physiological and pharmacological control [4], either on death rates rate $d_{i}$ or on phase transition rates $K_{i \rightarrow i+1}$ (possibly set to zero by effects of cytotoxic drugs). A typical cell cycle model reads

$$
\left\{\begin{array}{l}
\frac{\partial n_{i}(t, x)}{\partial t}+\frac{\partial\left\{v_{i}(x) n_{i}(t, x)\right\}}{\partial x}+d_{i}(t, x) n_{i}(t, x)+K_{i \rightarrow i+1}(t, x) n_{i}(t, x)=0, \\
n_{i+1}(t, 0)=\int_{0}^{\infty} K_{i \rightarrow i+1}(t, x) n_{i}(t, x) d x, \\
n_{1}(t, 0)=2 \int_{0}^{\infty} K_{I \rightarrow 1}(t, x) n_{I}(t, x) d x .
\end{array}\right.
$$

In a totally different setting, in an evolutionary dynamics representation, designed to describe the evolution of proliferating cell populations structured according to an evolutionary phenotype $x$, the dynamics of healthy $\left(n_{H}\right)$ and tumour $\left(n_{C}\right)$ cell populations undergoing a cytotoxic chemotherapy and subject to mutations may be written as

$$
\begin{aligned}
\frac{\partial}{\partial t} n_{H}(x, t)=[ & {[\overbrace{\frac{1-\theta_{H}}{(1+\rho(t))^{\beta}} r(x)}^{\text {growth with homeostasis }}-\overbrace{d(x)}^{\text {natural apoptosis }}-\overbrace{c(t) \mu_{H}(x)}^{\text {effect of drug }}] n_{H}(x, t)+\frac{\theta_{H}}{(1+\rho(t))^{\beta}} \underbrace{\int r(y) M_{\sigma_{H}}(y, x) n_{H}(y, t) d y}_{\text {birth with mutation }}, } \\
& \frac{\partial}{\partial t} n_{C}(x, t)=\left[\left(1-\theta_{C}\right) r(x)-d(x)-c(t) \mu_{C}(x)\right] n_{C}(x, t)+\theta_{C} \int r(y) M_{\sigma_{C}}(y, x) n_{C}(y, t) d y,
\end{aligned}
$$

where the total population is defined as

$$
\rho(t)=\rho_{H}(t)+\rho_{C}(t), \quad \rho_{H}(t)=\int_{x=0}^{\infty} n_{H}(x, t) d x, \quad \rho_{C}(t)=\int_{x=0}^{\infty} n_{C}(x, t) d x .
$$

In this setting, drug effects have been put on the death term $\mu(x)$ only (this holds for cytototoxic drugs). But as will be presented below, it is possible to include the representation of cytostatic drugs as acting on the proliferation rate $r(x)$. 


\section{DRUG EFFECTS IN HEALTHY AND CANCER CELL POPULATIONS}

\section{Modelling drug effects on the cell division cycle, healthy and cancer cell populations}

Solutions of linear models, such as McKendrick's, exhibit exponential growth. In Eq. 11), with time-periodic or stationary coefficients, solutions satisfy $n_{i}(t, x) \sim C^{0} N_{i}(t, x) e^{\lambda t}$ [2, 4], if $N_{i}$ are eigenvectors of the eigenvalue problem

$$
\left\{\begin{array}{l}
\frac{\partial N_{i}(t, x)}{\partial t}+\frac{\partial\left\{v_{i}(x) N_{i}(t, x)\right\}}{\partial x}+\lambda N_{i}(t, x)+d_{i}(t, x) N_{i}(t, x)+K_{i \rightarrow i+1}(t, x) N_{i}(t, x)=0, \\
N_{i+1}(t, 0)=\int_{0}^{\infty} K_{i \rightarrow i+1}(t, x) N_{i}(t, x) d x \\
N_{1}(t, 0)=2 \int_{0}^{\infty} K_{I \rightarrow 1}(t, x) N_{I}(t, x) d x \\
N_{i}>0, \quad N_{i}(t+T, .)=N_{i}(t, .), \quad \sum_{i} \int_{0}^{T} \int_{0}^{\infty} N_{i}(t, x) d x d t=1 .
\end{array}\right.
$$

with $T$-periodic coefficients to account for physiological (circadian) and pharmacological (periodically delivered) control. The study of the growth exponent $\lambda$, first eigenvalue of this positive growth system, is therefore crucial.
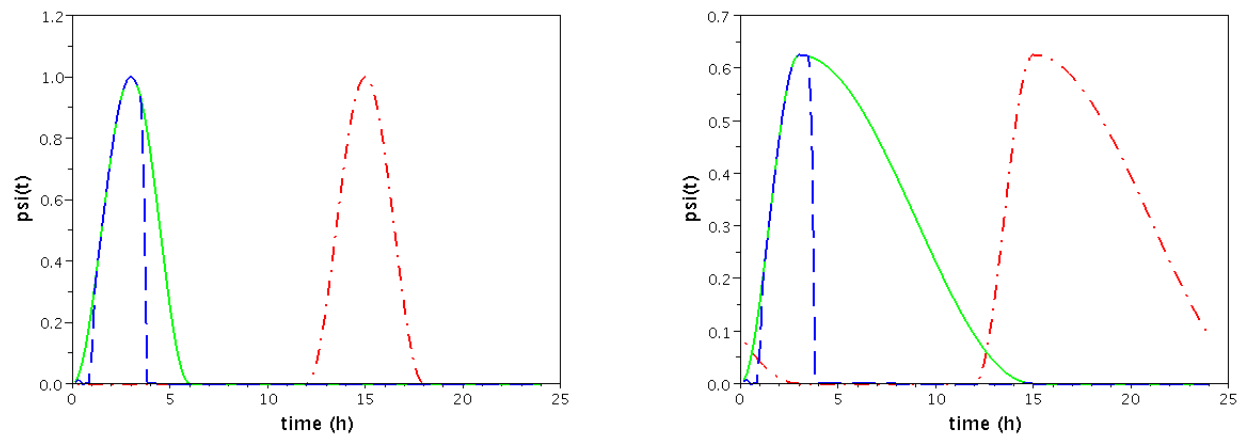

FIGURE 1. Periodic (circadian) phase transition gating rates $K_{i \rightarrow i+1}(t, x)$ at $G_{1} / S$ (red dash-pointed lines), at $G_{2} / M$ without drug (green full lines) and at $G_{2} / M$ with multiplication by a cytotoxic drug effect (dashed blue line). Left: healthy cells; right: cancer cells. The optimal drug infusion scheme consists in rectifying the 'lazy' cancer gating into a stiffer one, close to the healthy gating. The cytotoxic drug is assumed to be $S$ phase-specific, so that only the $G_{2} / M$ gating is affected by the drug. Redrawn from [4].
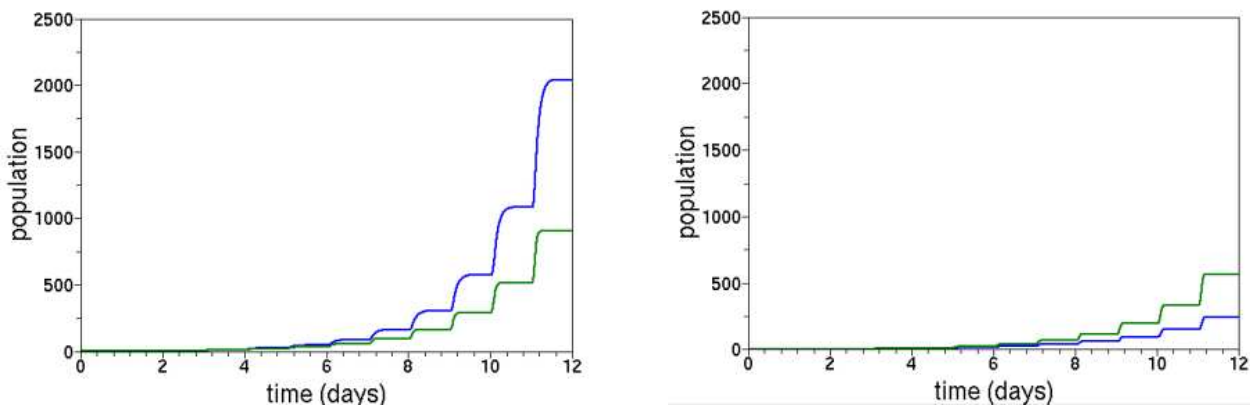

FIGURE 2. Effect of the drug on gating at $G_{2} / M$ transition. Left: without drug, the cancer cell population (blue line) outcompetes the healthy cell one (green line). Right: with partial drug blockade at $G_{2} / M$, both populations are hit, but the healthy cell population wins over the cancer cell one. Redrawn from [4].

In [4], we have shown how, taking transition rates $K_{i \rightarrow i+1}(t, x)$ as drug targets for a problem of optimal control by cytotoxic drugs on the eigenvalues $\lambda_{H}$ and $\lambda_{C}$, population growth exponents in healthy and cancer cell populations, one may numerically solve the optimal control problem, using a Uzawa-like algorithm. This is illustrated on Fig. 1 and 2. Moreover, possible targets for cytostatic drugs in this cell cycle setting are discussed in [5]; they include velocities $v_{i}(x)$ (otherwise usually set to 1 ), the boundary term in the first phase $\left(G_{1}\right)$ and the possible adjunction of a quiescent siding phase to which cells may escape at mitosis under the influence of a cytostatic drug, as in [6]. 


\section{Modelling drug-induced drug resistance in cancer cell populations}

In a setting slightly different from the general one presented above about evolutionary dynamics, we represent interactions of healthy and cancer cells separately with an environment variable. We assume that the cancer cell population is structured according to a 2-dimensional phenotype accounting for resistance to two categories of drugs: cytotoxic, acting on death rates, and cytostatic, acting on proliferation rates. Figures 3 and 4 show that cancer cell populations, that in the absence of drugs develop a non-resistant phenotype more favourable to proliferation, may in the presence of the two drugs show evolution towards two different resistant phenotypes simultaneously [5].

$$
\left\{\begin{array}{l}
\frac{\partial}{\partial t} n_{i}(t, x, y)=\overbrace{\left(r_{i}\left(x, y ; c_{\kappa \sigma}\right)-d_{i}(x, y) E_{i}(t)\right) n_{i}(t, x, y)}^{\text {growth with cytostatic drugs and death by competition }}-\overbrace{h_{i}\left(x, y ; c_{\kappa \tau}\right) n_{i}(t, x, y)}^{\text {effect of cytotoxic drugs }} \\
\frac{d}{d t} E_{i}(t)+\overbrace{\mu E_{i}(t)}^{\text {evolutionary environment }}=\overbrace{\sum_{j} a_{i j} \int_{0}^{1} \int_{0}^{1} n_{j}(t, x, y) d x d y}^{\text {interaction of cells with the environment }}
\end{array}\right.
$$
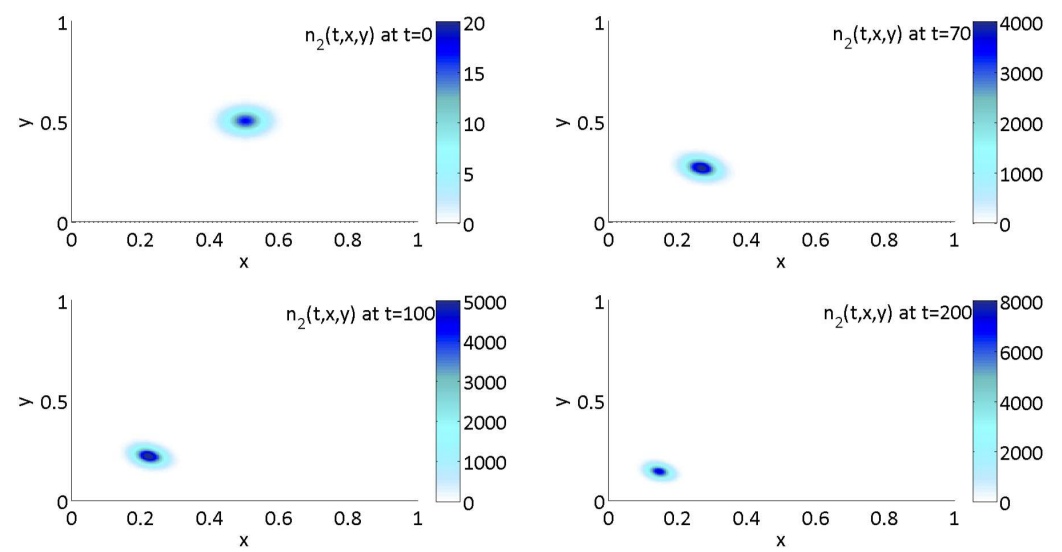

FIGURE 3. Cancer cells competing with healthy cells in the absence of drugs: evolution of the cancer cell population from an average phenotype $(x, y)=(0.5,0.5)$ towards sensitivity to both drugs, the cytostatic $\left(c_{\kappa \sigma}\right)$ and the cytotoxic $\left(c_{\kappa \tau}\right)$.
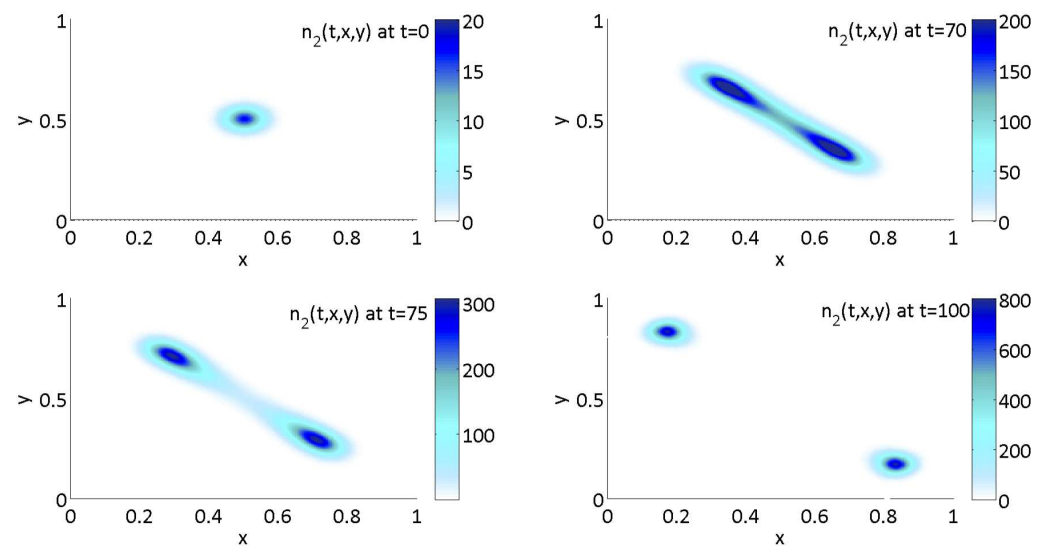

FIGURE 4. Cancer cells competing with healthy cells in the presence of both cytotoxic and cytostatic drugs: evolution of the cancer cell population towards two resistant subclones, one resistant to the cytotoxic, the other one resistant to the cytostatic drug.

In [7], we show how this setting, without necessary inclusion of mutations, allows to set the bases for a representation of combined cytostatic and cytotoxic anti-cancer therapies that overcomes the development of drug resistances, and may lead to extinction of the cancer cell population while preserving the healthy cell population. 


\section{CONCLUSION AND FUTURE PROSPECTS FOR THERAPEUTIC OPTIMISATION}

Within a general perspective of multidrug multiagent pharmacotherapeutic optimisation in oncology developed elsewhere [8, 9], we have focused here on two different types of proliferating cell population dynamics models with physiological structure, both with possible inclusion of targets for cytotoxic and for cytotoxic drugs separately. In the first one, we have shown how the representation of cytotoxic drug effects simultaneously in healthy and cancer cell populations leads to an optimal control problem with unwanted toxic side effects constraints, which is numerically solved in [4]; we note that with an accurate choice of targets for cytostatic drugs, it is possible to use the same numerical formalism to solve the same optimal control problem, using a combined cytotoxic-cytostatic therapy, as is frequently done in the clinic of cancers. In the second done, that belongs to the category of evolutionary dynamics models, already widespread in ecology but only recently developed in cancer biology [10], we present an adapted frame to theoretically tackle the problem of drug-induced drug resistance in cancer cell populations. We stress that in [7] is shown a first numerical attempt at representing a combined cytotoxic-cytostatic therapy, that not only leads to avoid drug resistance, but even more, yields extinction of the cancer cell population while preserving the healthy one from irreversible damage.

We have focused here on cytotoxic and cytostatic drugs, i.e., on treatments targeting cell death and proliferation. It is noteworthy that a third fate awaits proliferating cells, which is differentiation, that is, maturation towards fully functional state. A characteristic of cancer cells is their insufficiently differentiated status, which may be due to a blockade of differentiation at some stage of their maturation process, as is the case in acute myeloblastic leukaemia (AML). It is known in the case of acute promyelocytic leukaemia (APL, or AML3, a variety of AML in which differentiation is blocked at the promyelocyte stage) that a combination of cytotoxic drugs and redifferentiating agents cures the disease in $80 \%$ of patients. A rationale for the use of such a combined treatment with targets for the two types of drugs is presented in [11], following a previous model of AML [12]. We hypothesize, following this case, that designing models adapted to specific cancer diseases and targeting the three main fates of cancer cells, i.e., proliferation, apoptosis and differentiation, and solving optimal control problems under constraints of toxicity and/or drug resistance, may in the future help clinicians to propose successful treatments in cancers that hitherto remain out of reach of standard chemotherapies in the clinic.

\section{REFERENCES}

1. A. McKendrick, Proc. Edinburgh Math. Soc. 54, 98-130 (1926).

2. B. Perthame, Transport Equations in Biology, Frontiers in Mathematics series, Birkhäuser, Boston, 2007.

3. J. Clairambault, P. Michel, and B. Perthame, "A model of the cell cycle and its circadian control," in Mathematical Modeling of Biological Systems, Volume I: Cellular Biophysics, Regulatory Networks, Development, Biomedicine, and Data Analysis, edited by A. Deutsch, L. Brusch, H. Byrne, G. de Vries, and J. Herzel, Birkhäuser, Boston, 2007, pp. 239-251.

4. F. Billy, J. Clairambault, O. Fercoq, S. Gaubert, T. Lepoutre, T. Ouillon, and S. Saito, Math. Comp. Simul. (2012), in press, available on line Apr. 2012.

5. F. Billy, and J. Clairambault, Designing proliferating cell population models with functional targets for control by anti-cancer drugs (2012), submitted.

6. P. Gabriel, S. P. Garbett, D. R. Tyson, and G. F. Webb, The contribution of age structure to cell population responses to targeted therapeutics (2011), Journal of Theoretical Biology, in revision, 2012. First version downloadable from http://hal.archives-ouvertes.fr/hal-00649178.

7. A. Lorz, T. Lorenzi, M. Hochberg, J. Clairambault, and B. Perthame, Populational adaptive evolution, chemotherapeutic resistance and multiple anti-cancer therapies (2012), Mathematical Modelling and Numerical Analysis, accepted, http://hal.archives-ouvertes.fr/hal-00714274.

8. J. Clairambault, Mathematical Modelling of Natural Phenomena 4, 12-67 (2009).

9. J. Clairambault, Personalized Medicine 8, 271-286 (2011).

10. M. Deliltala, and T. Lorenzi, Journal of Mathematical Analysis and Applications 389, 439-451 (2012).

11. H. Özbay, C. Bonnet, H. Benjelloun, and J. Clairambault, Mathematical Modelling of Natural Phenomena 7, 203-234 (2012).

12. M. Adimy, F. Crauste, and A. El Abdllaoui, Journal of Biological Systems 16, 395-424 (2008). 